\title{
Mean Platelet Volume and Mean Platelet Volume/Platelet Count Ratio in Chronic Urticaria
}

\author{
June Hyuck Yim, Hyung Jin Park, Sun Young $\mathrm{Cho}^{1}$, Min Kyung Shin \\ Departments of Dermatology and ${ }^{1}$ Laboratory Medicine, College of Medicine, Kyung Hee University, Seoul, Korea
}

\begin{abstract}
Dear Editor:
Chronic urticaria (CU) is characterized by recurrent transitory, itchy wheals that last for a period of at least 6 weeks ${ }^{1}$. Recently, platelets have been recognized as playing a vital role in immune and inflammatory reactions in several inflammatory cutaneous disorders such as allergic dermatitis, atopic dermatitis, psoriasis, and urticaria ${ }^{2}$. Mean platelet volume (MPV) is a useful laboratory index for estimating platelet function and activation and is frequently used as an inflammatory marker in various diseases associated with inflammation. However, the role of MPV in patients with CU remains controversial. ConfinoCohen et al. ${ }^{3}$ described significantly higher MPV values in $12,778 \mathrm{CU}$ patients compared with 10,714 control subjects, whereas Isiksacan et al. ${ }^{4}$ reported MPV is significantly decreased in $\mathrm{CU}$ patients. Therefore, in the present study we compared the MPV between patients with and without $\mathrm{CU}$.

Patients who presented to the dermatologic clinic of Kyung Hee University between January 2012 and December 2014 for CU were retrospectively enrolled. This study was approved by the Institutional Review Board (IRB) of Kyung Hee University Medical Center (approval number: KHUHMDIRB 2017-12-075). CU was defined as urticarial symptoms lasting 6 or more weeks. The control group consisted of 143 individuals selected randomly from those who underwent medical check-ups at the same hospital. Cases and controls with other diseases which could influence platelet level such as hypertension, hyperlipidemia, diabetes mellitus, metabolic syndrome, and fatty liver dis-
\end{abstract}

ease, as well as patients taking any medication other than antihistamines for 3 months prior to enrollment were excluded from the study. Blood sampling was performed at the initial visit and the MPV was measured within 2 hours using an Advia 2120 (Siemens Healthcare Diagnostics, Tarrytown, NY, USA). The measured parameters included platelet count, MPV and MPV/plate count ratio. Thyroidstimulating hormone, thyroglobulin antibodies, and thyroperoxidase were evaluated in $\mathrm{CU}$ patients, and patients with abnormal findings in those laboratory tests were classified as chronic autoimmune-related urticaria (CAU), while others as chronic idiopathic urticaria (CIU). Data were analyzed using SPSS ver. 12.0 (SPSS Inc., Chicago, IL, USA). The Mann-Whitney $U$ test was used to compare data between patient groups and healthy controls and analysis of covariance (ANCOVA) controlling for sex and age was used to exclude the differences due to these variables. $p$-values $<0.05$ were considered statistically significant.

A total of 292 patients with CU (82 males; mean age, $36.47 \pm 16.15$ years) and 143 healthy patients ( 72 males; mean age, $44.00 \pm 10.37$ years) were enrolled (Table 1). Platelet count was not significantly different between the two groups. However, significant differences were observed in MPV and the MPV/platelet count ratio. MPV was significantly lower in $\mathrm{CU}$ patients compared with the controls $(7.45 \pm 0.69$ vs. $7.96 \pm 0.58 \mathrm{fl}, p=0.000)$. In addition, the MPV/platelet count ratio was significantly lower in the patient group compared with the control group $(0.030 \pm$ 0.009 vs. $\left.0.032 \pm 0.00710^{9} / \mathrm{L}, p=0.001\right)$. Because this

Received January 10, 2018, Revised August 6, 2018, Accepted for publication August 15, 2018

Corresponding author: Min Kyung Shin, Department of Dermatology, College of Medicine, Kyung Hee University, 23 Kyungheedae-ro, Dongdaemun-gu, Seoul 02447, Korea. Tel: 82-2-958-8300, Fax: 82-2-969-6538, E-mail: haddal@hanmail.net ORCID: https://orcid.org/0000-0001-9834-7931

This is an Open Access article distributed under the terms of the Creative Commons Attribution Non-Commercial License (http://creativecommons.org/ licenses/by-nc/4.0) which permits unrestricted non-commercial use, distribution, and reproduction in any medium, provided the original work is properly cited.

Copyright (C) The Korean Dermatological Association and The Korean Society for Investigative Dermatology 
Table 1. Characteristics of patients with chronic urticaria (CU), chronic idiopathic urticaria (CIU), chronic autoimmune-related urticaria $(\mathrm{CAU})$ and the control group

\begin{tabular}{|c|c|c|c|}
\hline & \multicolumn{2}{|c|}{$\mathrm{CU}(\mathrm{n}=292)$} & \multirow{2}{*}{$\begin{array}{l}\text { Control } \\
(n=143)\end{array}$} \\
\hline & CIU $(n=207)$ & CAU $(n=85)$ & \\
\hline Mean age (yr)* & $36.36 \pm 16.06$ & $36.74 \pm 16.44$ & $44.00 \pm 10.37$ \\
\hline $\begin{array}{l}\text { Sex } \\
\text { (male:female)* }\end{array}$ & $82: 125$ & $30: 55$ & $72: 71$ \\
\hline $\begin{array}{l}\text { Platelet count } \\
\left(10^{9} / \mathrm{L}\right)\end{array}$ & $263.91 \pm 65.23$ & $266.57 \pm 64.89$ & $257.95 \pm 49.2$ \\
\hline $\begin{array}{l}\text { Mean platelet } \\
\text { volume }(\mathrm{fl})^{*,}\end{array}$ & $7.48 \pm 0.71$ & $7.36 \pm 0.65$ & $7.96 \pm 0.58$ \\
\hline $\begin{array}{l}\text { MPV/platelet } \\
\text { count ratio } \\
\left(10^{6} / \mathrm{L}\right)^{*}\end{array}$ & $30.22 \pm 8.60$ & $29.53 \pm 9.29$ & $32.09 \pm 7.21$ \\
\hline
\end{tabular}

Values are presented as mean \pm standard deviation or number only. ${ }^{*}$ This parameter showed statistically significant differences in all cases except for the comparison between the CIU group and CAU group. ${ }^{\dagger}$ This parameter showed statistically significant differences after controlling sex and age in all comparisons except for the analysis between the CIU group and CAU group.

was not a sex- and age-matched study, sex and age were significantly different between $\mathrm{CU}$ groups and control group ( $p=0.018$ for sex, $p=0.000$ for age). Therefore, ANCOVA was additionally performed and results showed the difference in MPV remained significant $(p=0.000)$, but the difference in the MPV/platelet ratio was not statistically significant $(p=0.118)$.

The association between $\mathrm{CU}$ and autoimmune thyroid disease is generally well recognized. Likewise, the association between thyroid disease and $\mathrm{CU}$ is sufficiently strong. Therefore, we compared the parameters of patients in the $\mathrm{CAU}$ group with the other groups (CIU and control). A total of 85 patients had an abnormal thyroid examination. The MPV and MPV/platelet ratio were both significantly lower in the $\mathrm{CU}, \mathrm{CIU}$, and $\mathrm{CAU}$ groups than the control group (for MPV, $p=0.000,0.000$, and 0.000 , respectively; for MPV/platelet count ratio, $p=0.002,0.014$, and 0.001 , respectively). However, after controlling the effect of sex and age using ANCOVA, differences in MPV/platelet count ratio were no longer significant $(p=0.118, p=$ 0.211 , and $p=0.124$, respectively). However, MPV remained lower in the $\mathrm{CU}, \mathrm{CIU}$, and $\mathrm{CAU}$ groups after controlling for sex and age $(p=0.000,0.000$, and 0.000 , respectively). In the analysis between the CIU group and the $\mathrm{CAU}$ group, there were no statistical differences in platelet, MPV, MPV/platelet count ratio $(p=0.519,0.088$, and 0.172 , respectively).

MPV has been investigated in a diverse range of diseases, including cardiovascular disease, rheumatoid arthritis, and
Alzheimer's disease ${ }^{5}$. Among the cutaneous disorders, psoriasis, atopic eczema, and systemic lupus erythematosus (SLE) are associated with MPV. Researchers reported that MPV is increased in those diseases and correlated with disease severity in psoriasis and $\mathrm{SLE}^{5-7}$.

The results from existing studies on the relationship between MPV and CU are controversial. Gasparyan et al. ${ }^{8}$ provided an explanation for these contradictory findings, hypothesizing that high-grade inflammatory diseases result in a low MPV, while low-grade inflammatory diseases have the opposite effect on MPV. Based on these studies, the nature of MPV as an inflammatory marker remains controversial, and thus cannot be generalized at present. The present study had some limitations. First, we did not attempt to correlate MPV or MPV/platelet ratio with disease duration, severity, or response to therapy. Based on the study design, we could not investigate the above topics. Second, the entire population of CAU patients may not have been adequately covered in the present study. Confino-Cohen et al. ${ }^{3}$ reported that autoimmune diseases, which were mostly autoimmune thyroid disorders, were significantly more common in patients with $\mathrm{CU}$ than in control patients. Other autoimmune diseases such as rheumatoid arthritis, Sjögren syndrome, celiac disease, type I diabetes mellitus, and SLE were also more common in CU patients, but only significant in female patients, and were mostly diagnosed during the 10 years after the diagnosis of $\mathrm{CU}$. Therefore, thyroid disease may be present in most CAU patients, however, this study may reflect only a portion of the CAU population.

In conclusion, MPV and MPV/platelet count ratios were significantly lower in $\mathrm{CU}$ patients compared with healthy controls. This result was consistent for all CU patients regardless of disease subtype, namely, CIU or CAU as determined based on thyroid studies. Taken together, our results indicate that MPV, which can be included in routine laboratory tests, may be lower in patients with $\mathrm{CU}$, and it can be regarded as a trail of $\mathrm{CU}$.

\section{CONFLICTS OF INTEREST}

The authors have nothing to disclose.

\section{ORCID}

June Hyuck Yim, https://orcid.org/0000-0002-5043-155X Hyung Jin Park, https://orcid.org/0000-0002-5952-9158 Sun Young Cho, https://orcid.org/0000-0002-3208-5446 Min Kyung Shin, https://orcid.org/0000-0001-9834-7931 


\section{REFERENCES}

1. Akelma AZ, Mete E, Cizmeci MN, Kanburoglu MK, Malli DD, Bozkaya D. The role of mean platelet volume as an inflammatory marker in children with chronic spontaneous urticaria. Allergol Immunopathol (Madr) 2015;43:10-13.

2. Chandrashekar L, Rajappa M, Sundar I, Munisamy M, Ananthanarayanan $\mathrm{PH}$, Thappa DM, et al. Platelet activation in chronic urticaria and its correlation with disease severity. Platelets 2014;25:162-165.

3. Confino-Cohen R, Chodick G, Shalev V, Leshno M, Kimhi $\mathrm{O}$, Goldberg A. Chronic urticaria and autoimmunity: associations found in a large population study. J Allergy Clin Immunol 2012;129:1307-1313.

4. Isiksacan N, Koser M, Cemsitoglu F, Kucuksezer UC, Gurdol F. Platelet and other hemostatic characteristics in patients with chronic urticaria. Angiology 2015;66:387-391.

5. Kim DS, Lee J, Kim SH, Kim SM, Lee MG. Mean platelet volume is elevated in patients with psoriasis vulgaris. Yonsei Med J 2015;56:712-718.

6. Topal E, Celiksoy MH, Catal F, Karakoç HT, Karadağ A, Sancak $\mathrm{R}$. The platelet parameters as inflammatory markers in preschool children with atopic eczema. Clin Lab 2015; 61:493-496.

7. Bai M, Xing L, Feng J, Cui C, Huang L, Liang G. Mean platelet volume could reflect disease activity of adult patients with systemic lupus erythematosus. Clin Lab 2016;62: 1317-1322.

8. Gasparyan AY, Ayvazyan L, Mikhailidis DP, Kitas GD. Mean platelet volume: a link between thrombosis and inflammation? Curr Pharm Des 2011;17:47-58.

\title{
Comparative Study of Clinical Characteristics according to Therapeutic Efficacy and Drug Survival of Cyclosporine or Methotrexate in Psoriasis Vulgaris
}

\author{
Hyun Soo Lee, Soo Hyun Kwon, Eun-So Lee \\ Department of Dermatology, Ajou University School of Medicine, Suwon, Korea
}

Dear Editor:

Psoriasis vulgaris is a chronic, multifactorial inflammatory skin disease. Cyclosporine (CsA) and methotrexate (MTX) are conventional but still highly effective therapeutic options for patients with psoriasis vulgaris, as not all patients are appropriate for biological therapy.

Since the introduction of the tumor necrosis factor (TNF) inhibitors and other biological agents for the treatment of psoriasis, CsA and MTX have become less frequently discussed $^{1}$. In particular, no studies have focused on the clinical and laboratory factors to predict therapeutic response to CsA or MTX. This study aimed to compare clinical and laboratory characteristics according to therapeutic response to either CsA or MTX. Furthermore, we aimed to demonstrate the predictive factors of therapeutic response to either CsA or MTX.

\section{Received July 23, 2018, Revised October 10, 2018, Accepted for publication October 26, 2018}

Corresponding author: Eun-So Lee, Department of Dermatology, Ajou University School of Medicine, 164 WorldCup-ro, Yeongtong-gu, Suwon 16499, Korea. Tel: 82-31-219-5190, Fax: 82-31-219-5189, E-mail: esl@ajou.ac.kr ORCID: https://orcid.org/0000-0003-0232-7704

This is an Open Access article distributed under the terms of the Creative Commons Attribution Non-Commercial License (http://creativecommons.org/ licenses/by-nc/4.0) which permits unrestricted non-commercial use, distribution, and reproduction in any medium, provided the original work is properly cited.

Copyright (C) The Korean Dermatological Association and The Korean Society for Investigative Dermatology 